\title{
Acute heart failure and complete AV block in a patient with severe Covid-19 pneumonia.
}

\author{
Hugo Rodriguez-Zanella ${ }^{1}$, Alan Gutierrez-Villaseñor ${ }^{2}$, Juan Pablo Romero-Gonzalez ${ }^{2}$, \\ Adriana Torres-Machorro ${ }^{1}$, Ana Tobias ${ }^{1}$, and Silvio Namendys-Silva ${ }^{3}$ \\ ${ }^{1}$ National Institute of Cardiology of Mexico \\ ${ }^{2}$ Medica Sur \\ ${ }^{3}$ Instituto Nacional de Cancerologia
}

August 12, 2020

\begin{abstract}
We present the challenging case of a patient with severe COVID-19 pneumonia complicated with cardiogenic shock and refractory complete AV block. The patient presented with wall motion abnormalities on echocardiography, and cardiac magnetic resonance revealed finding compatible with myocarditis. Myocardial injury may occur in up to $31 \%$ of patients with Covid-19 pneumonia and has been related to increased mortality. However, whether myocardial injury might result from direct damage from the virus or related to complications of the disease is still unclear. Although myocarditis is not common in COVID patients, it should be considered as a possible diagnosis.
\end{abstract}

Acute heart failure and complete AV block in a patient with severe Covid-19 pneumonia.

Authors.

Rodríguez-Zanella Hugo MD

Echocardiography Department

National Institute of Cardiology of Mexico, Ignacio Chavez

Gutiérrez-Villaseñor Alan O.MD

Intensive care Unit, Medica Sur, Clinical Foundation

Institution: Medica Sur, Clinical Foundation

Romero-González Juan P. MD

Intensive care Unit, Medica Sur, Clinical Foundation

Adriana Torres-Machorro MD

Surgery Department

National Institute of Cardiology of Mexico, Ignacio Chavez

Ana Karla Tobias Morales MD

Echocardiography Department

National Institute of Cardiology of Mexico, Ignacio Chavez 
Corresponding Author.

Silvio A, ÑamendysSilva MD, MSc, FCCP,FCCM

Address: Instituto Nacional de Cancerologia and Instituto Nacional de Ciencias Medicas y Nutricion Salvador Zubiran, 14080, Mexico City, Mexico

Email:snamendys@incan.edu.mx

Contact: +525556280400

Abstract

We present the challenging case of a patient with severe COVID-19 pneumonia complicated with cardiogenic shock and refractory complete AV block. The patient presented with wall motion abnormalities on echocardiography, and cardiac magnetic resonance revealed finding compatible with myocarditis. Myocardial injury may occur in up to $31 \%$ of patients with Covid-19 pneumonia and has been related to increased mortality. However, whether myocardial injury might result from direct damage from the virus or related to complications of the disease is still unclear. Although myocarditis is not common in COVID patients, it should be considered as a possible diagnosis.

Keywords: Cardiomyopathies, Heart Failure, Echocardiography, Magnetic Resonance

We describe a patient with severe COVID pneumonia, with acute heart failure, cardiogenic shock, and complete AV Block in a critical care unit designated to treat Covid-19 patients. Myocardial injury may occur in up to $31 \%$ of patients with Covid-19 pneumonia and has been related to increased mortality $(1,2)$. Whether myocardial injury might result from direct damage from the virus or related to complications of the disease is still unclear.

A 65 years old male with a history of diabetes mellitus, presented to the emergency department with shortness of breath, fever, cough, and low oxygen saturation (SPO2 87\%). Thoracic CT was performed, demonstrating typical Covid-19 findings. (Figure 1 panel C) Confirmatory RT-PCR and diagnosis of Severe acute respiratory syndrome coronavirus 2 (SARS-CoV-2) was made on April 20. PCR testing for other respiratory viruses was negative. Symptomatic treatment and thromboprophylaxis with low molecular weight heparin were instaurated.

Two days later, the patient progressed to hypoxemic respiratory failure requiring mechanical ventilation. The patient was admitted to the intensive care unit, hemodynamically stable, and responded well to mechanical ventilation with lung protection. The patient was doing better, and weaning protocol was already planned. However, on April 30, the patient suddenly presented with hypotension and bradycardia. A surface ECG showed complete AV Block, with left bundle branch block (Figure 1 panel A). The patient was started on vasopressors, and a temporary pacemaker was placed. Initial high sensitivity troponin was elevated (1730 $\mathrm{ng} / \mathrm{ml}$ ), and BNP was normal, left ventricular function was reduced, and wall motion abnormalities in the apical segments were found (Figure 1 panel D and Video 1).

Differentials included Takotsubo cardiomyopathy, acute myocarditis, and acute coronary syndrome. Because of the previous risk factors and elevated high sensitivity troponin I level, coronary angiography was performed. Significant proximal stenosis in the left anterior descending artery was found. Percutaneous coronary intervention with a drug-eluting stent was performed in the context of cardiogenic shock. The patient improved progressively with the aid of levosimendan. Vasopressors and temporary pacemaker were successfully removed, and the patient was extubated 8 days later.

Cardiac magnetic resonance (CMR) showed reduced left ventricular ejection fraction (LVEF 27\%) and subepicardial late gadolinium enhancement in the inferior septum and anterior wall (Figure 1 panel E and F), compatible with subacute myocarditis (videos 2 and 3). Latter findings made ischemic or Takotsubo cardiomyopathy unlikely. A few days later, he had hypotension and new onset complete AV block with right bundle branch block and ST-segment depression in V1-V3 (Figure 1 panel B). Coronary angiography was 
performed to ensure stent patency and definitive pacemaker was placed. He was started in guideline directed medical treatment for heart failure. Endomyocardial biopsy was not performed due to clinical improvement and increased s risk to health care providers. A few days later, he was discharged. On follow up one month later left ventricular function recovered.

Myocarditis is a well-described feature of other viral illnesses; however, in patients with SARS-CoV-2, data is limited to a few case reports. Although myocardial injury is common in patients with severe disease. Histopathological studies have only shown a few interstitial mononuclear inflammatory infiltrates in the myocardium (3). Cardiac magnetic resonance is a valuable tool for myocardial tissue characterization in vivo validated against histology in a wide range of clinical scenarios. Interestingly in patients with moderate to severe SARS-CoV-2 disease, CMR can detect late gadolinium enhancement (LGE) in half of recovered patients who reported chest pain or palpitations (4). Thus, myocarditis might be more common than expected.

Complete AV block in patients with SARS-CoV-2 myocarditis might occur. The mechanism involved is yet to be defined. The high expression of Angiotensin-converting enzyme 2 in heart and vessels might pose these tissues as primary target of the disease. A better understanding of cardiac complications of SARS-Cov2 infection is needed to guide clinicians in the treatment of these patients.

Author contributions

Rodríguez-Zanella Hugo MD , Care of the patient, Conceptualization, Writing original draft, Writing- review and editing, Approval

Gutiérrez-Villaseñor Alan O.MD, Care of the patient, Data Curation, review and editing, Approval

Romero-González Juan P. MD, Care of the patient, Data Curation, review and editing, Approval

Adriana Torres-Machorro MD Care of the patient, Data Curation, review and editing, Approval

Ana Karla Tobias Morales MD, Care of the patient, Data Curation, Writing- review and editing, Approval

Silvio A, Namendys Silva MD, MSc, FCCP,FCCM Care of the patient, Conceptualization, Writing- review and editing,

References.

1. Shi S Shi S, Qin M, Shen B, et al. Association of cardiac injury with mortality in hospitalized patients with COVID-19 in Wuhan, China. JAMA Cardiol 2020 March 25 (Epub ahead of print)

2. Guo T, Fan Y, Chen M, Wu X, Zhang L, He T, Wang H, Wan J, Wang X, Lu Z. Cardiovascular implications of fatal outcomes of patients with coronavirus disease 2019 (COVID-19). JAMA Cardiol 2020 March 27 (Epub ahead of print).

3. Xu Z, Shi L, Wang Y, et al. Pathological findings of COVID-19 associated with acute respiratory distress syndrome. Lancet Respir Med 2020 Apr;8(4):420-422.

4. Huang L, Zhao P, Dazhong T, et al. Cardiac involvement in recovered COVID-19 patients identified by magnetic resonance imaging. J Am Coll Cardiol Img. 2020 May 11. (Epub ahead of print)

Figure 1. Representative images during the patient hospital stay. Panel A. 12 lead surface ECG with complete AV block and left bundle branch block at day 12 from admission. Panel B. 12 lead ECG with complete AV block and right bundle branch block. Panel C. CT showing bilateral interstitial infiltrates typical of Covid 19 pneumonia (arrow). Panel D. Four chamber echocardiographic view showing apical dyskinesia (arrow). Panel E. Cardiac magnetic resonance cine image, short axis view with normal wall thickness. Panel F. Late gadolinium enhancement CMR showing subepicardial enhancement in the inferior septum. LA= left atrium, $\mathrm{LV}=$ left ventricle, $\mathrm{RA}=$ right atrium, $\mathrm{RV}$ right ventricle.

Video 1. Apical four chamber view, showing apical dyskinesia, and normal wall motion of the basal segments. $\mathrm{LA}=$ left atrium, $\mathrm{LV}=$ left ventricle, 
$\mathrm{RA}=$ right atrium, RV right ventricle. Video 2. CMR short axis view comparative. A. Cine, (SSFP) showing reduced left ventricular function B. T1 weighted sequence showing normal pericardium. C. Short time recovery image, showing no edema. D. Late gadolinium enhancement imaging, showing epicardial anterior and intramyocardial inferoseptal enhancement

$\mathrm{LV}=$ left ventricle, RV right ventricle. Video 3. CMR cine images in two, three and four chamber view showing reduced left ventricular function, and wall motion abnormalities in the apical segments. LA= left atrium, $\mathrm{LV}=$ left ventricle, $\mathrm{LVOT}=$ left ventricular outflow tract, $\mathrm{RA}=$ right atrium, $\mathrm{RV}$ right ventricle.

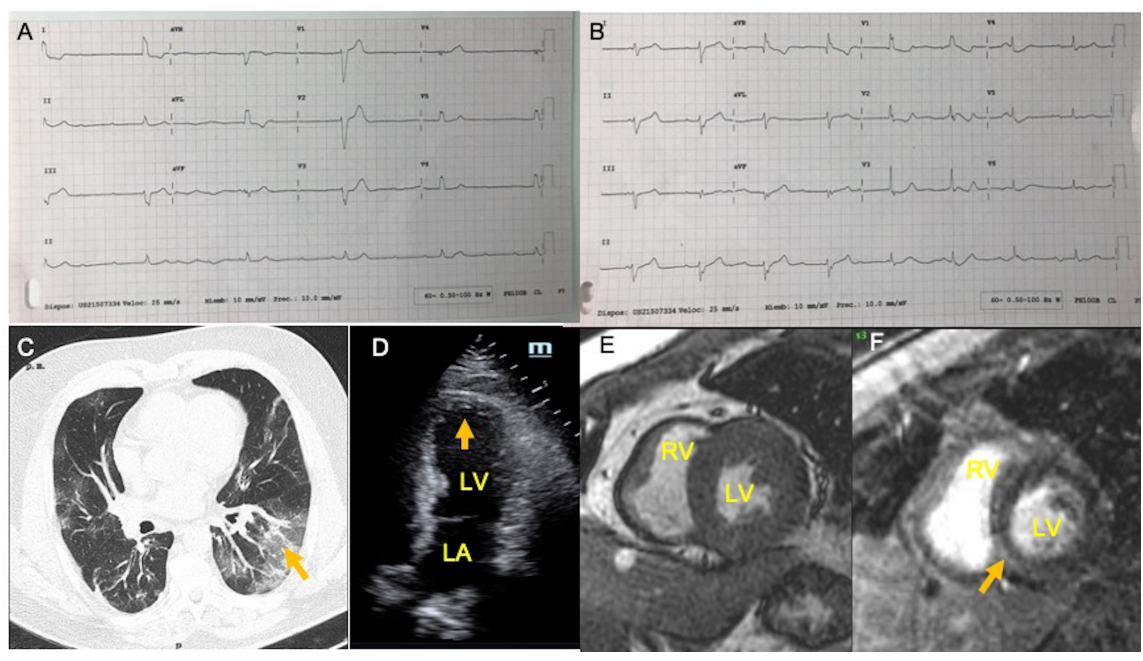

